\title{
Dante, Ulisse e la scrittura della nuova epica: una lettura del Canto XXVI dell'Inferno
}

\author{
Mario D'Alessandro
}

Nel famoso dibattito Nardi-Fubini sulla figura dantesca di Ulisse si concentrano quei due poli opposti che hanno per tanti anni determinato le prese di posizione sull'argomento. Se Bruno Nardi vede Ulisse come rappresentazione sostanzialmente negativa del primo peccato umano, ${ }^{1}$ Mario Fubini, respingendo ogni interpretazione moderna dell'episodio, ${ }^{2}$ sostiene invece il valore positivo della visione dantesca dell'eroe pagano: "l'impresa [di Ulisse], [...] da null'altro spinto che dall'amore del vero, [...] ci sembra quasi un simbolo per eccellenza di quel che più nobile è nell'umanità pagana o più generalmente nell 'umanità ignara della rivelazione" (469-70). Il parere di Rocco Montano, pur rientrando nel campo dei giudizi più o meno negativi sulla figura di Ulisse, è un tentativo di moderare gli eccessi positivi del Fubini: ${ }^{3}$ per Montano, l'Ulisse dantesco non rappresenta altro che "un vano e distorto ricercare, di una sete di conoscenza che per il poeta, come per tutto il mondo medievale, era curiositas, peccato, prostitutio nostrae virtutis rationalis" (175). Tuttavia, il giudizio del Montano, come gli altri qui citati, rientra nello stretto ambito di un'analisi teologico-morale. Questo non significa negare né la validità dell'esegesi medioevale né il poetare allegorico di Dante: significa solo che l'interpretazione del Montano, come quella del Nardi e del Fubini, non spiega in modo sufficientemente ampio il significato dell'incontro fra Ulisse e Dante-pellegrino, il primo legato a un mondo e forse anche a una visione gnoseologica ormai scomparsi mentre l'altro si trova sulla soglia di un nuovo mondo non ancora scritto e di cui manca la massima espressione poetica. ${ }^{4}$ Come ha intuito solo in parte la critica dantesca americana, l'incontro fra Ulisse e Dante-pellegrino è carico di valore drammatico non ancora ben chiarito, ed è proprio qui, nel tessuto poetico stesso dell'incontro e del contrasto fra $i$ due personaggi e non nel giudizio teologico di Dante-poeta o in quello poetico di Dante-pellegrino, che si manifesta un'altra strada interpretativa da seguire.

Il critico americano John Freccero, in un suo saggio intitolato "Dante's Ulysses: From Epic to Novel", scrive del primo contatto fra Virgilio e Ulisse: "Naive commentators have attempted to gloss this passage [Inf. 26.73-74] by saying that it means simply that Virgil could understand Greek, while the 
poet [Dante] could not" (141). Dire tuttavia che il linguaggio che Virgilio e Ulisse hanno in comune "is [...] the high style of ancient epic, whose qualities are unappreciated in the vulgar company of Hell, where the language is the sermo humilis of Christian comedia", come asserisce Freccero (141), non spiega bene perché dalla loro fiamma gli eroi greci, Ulisse e Diomede, "sarebbero schivi" del "detto" (Inf. 26.74-75) di Dante. ${ }^{5}$ A mio modo di vedere, Freccero non individua il vero motivo della scissione linguistica fra Dante e Ulisse, fra stile umile e stile epico, fra mondo medievale e mondo classico, che va ricercato invece nelle prime pagine del De Vulgari Eloquentia.

Opera stesa fra il 1303 e il 1305, il De Vulgari Eloquentia è quel trattato in latino in cui Dante, mentre ancora scriveva la Commedia, cercò di teorizzare l'eloquenza, ovvero il parlare, volgare:

Volentes discretionem aliquiter lucidare illorum qui tanquam ceci ambulant per plateas, plerunque anteriora posteriora putantes, Verbo aspirante de celis locutioni vulgarium gentium prodesse temptabimus, [...]. (1.1.1)

[Volendo in qualche modo illuminare il discernimento di coloro che come ciechi si aggirano per le piazze, per lo più credendo di avere davanti ciò che invece hanno dietro, con la grazia del Verbo che spira dal cielo, cercherò di giovare alla lingua delle genti volgari. $]^{6}$

Dante definisce il volgare come quella lingua che i bambini imparano senza regole da chi sta loro intorno:

Vulgarem locutionem appellamus eam qua infantes assuefiunt ab assistentibus cum primitus distinguere voces incipiunt; vel, quod brevius dici potest, vulgarem locutionem asserimus quam sine omni regula nutricem imitantes accipimus. (1.1.2)

[Chiamo lingua volgare quella che i bambini apprendono da chi sta loro intorno dal momento che cominciano ad articolare i suoni; oppure, per essere più brevi, lingua volgare è quella che, senza bisogno di regole, impariamo imitando la nostra nutrice.]

Il volgare si distingue da quella se conda lingua che i Romani chiamarono grammatica, ${ }^{7}$ e che, a differenza del volgare, è la lingua dei pochi: "Ad habitum vero huius pauci perveniunt, quia non nisi per spatium temporis et studii assiduitatem regulamur et doctrinamur in illa" (1.1.3) ["Ad avere familiarità con essa, per la verità, ci riescono in pochi, perché solo con molta disponibilità di tempo e assiduità di studio si possono apprenderne regole e arte"]. La più nobile fra queste due lingue è il volgare perché "prima fuit humano generi usitata; tam quia totus orbis ipsa perfruitur, licet in diversas prolationes et vocabula sit divisa" (1.1.4) ["di queste due, la volgare è più nobile: e perché fu per prima usata dal genere umano e perché se ne serve tutto il mondo, ancorché sia divisa in differenti pronunce e vocaboli"]. ${ }^{8}$ Il volgare ha precedenza sulla grammatica non solo perché è lingua universale in quanto lingua di tutti, ma anche perché ha precedenza cronologica: "Hec est nostra vera prima 
locutio" (2.1.1). La precedenza cronologica, o meglio mito-cronologica, del volgare indica anche, come vedremo, la sua precedenza logica e quindi gnoseologica nei confronti della grammatica.

L'importanza che Dante dà qui alla lingua indica una chiara preferenza per la lingua e non la ragione in senso astratto ${ }^{9}$ come la vera caratteristica che distingue l'uomo sia dagli angeli e i demoni (1.2.3-4) che dagli animali (1.2.5):

[...] soli homini datum est loqui, cum solum sibi necessarium fuerit. Non angelis, non inferioribus animalibus necessarium fuit loqui, sed nequicquam datum fuisset eis: quod nempe facere natura aborret. (1.2.2)

[Tra tutti gli esseri viventi solo all'uomo fu dato di parlare, perché solo a lui fu necessario. Mentre non lo fu né per gli angeli né per gli animali inferiori, cui la parola sarebbe stata data inutilmente: cosa che natura evita accuratamente di fare.]

Dato che gli umini "nec per spiritualem speculationem, ut angelum, alterum alterum introire contingit" (1.3.1) ["né gli uomini si possono reciprocamente compenetrare attraverso un rispecchiamento spirituale, come capita agli angeli. perché lo spirito umano è impacciato dalla grossezza e opacità del corpo mortale"], fu necessario che l'uomo "ad comunicandas inter se conceptiones suas aliquod rationale signum et sensuale habere" (1.3.2) ["fu dunque necessario che il genere umano, per comunicarsi i propri concetti, avesse un segno insieme razionale e sensibile"]. Il concetto dantesco del signum è interessante proprio perché Dante lo vede come unità armonica fra la ragione e i sensi: ${ }^{10}$

Quia, cum de ratione accipere habeat et in rationem portare, rationale esse oportuit; cumque de una ratione in aliam nichil deferri possit nisi per medium sensuale, sensuale esse opportuit. (1.3.2)

[Poiché infatti si tratta di ricevere dalla ragione e di portare alla ragione, doveva essere razionale; e poiché da una ragione all'altra non può passare nulla se non per un mezzo sensibile, doveva essere sensibile.]

Il signum che Dante esplica e definisce è infatti l'argomento stesso del trattato:

Hoc equidem signum est ipsum subiectum nobile de quo loquimur: nam sensuale quid est in quantum sonus est; rationale vero in quantum aliquid significare videtur ad placitum. (1.3.3)

[E proprio questo segno quel nobile argomento di cui ci stiamo occupando: esso, infatti, è sensibile in quanto è suono; è razionale invece in quanto pare significare qualcosa a nostro arbitrio.]

Il signum dantesco è un 'insieme armonico fra suono e ragione, cioè fra la poesia e l'atto interpretativo del "suono" poetico. Se il "suono" è l'enunciato poe- 
tico nella sua piena complessità fonica , la ragione è quel "pan de li angeli" del Trattato Primo del Convivio (1.1.7) e del Canto 2 del Paradiso (Par. 2.11). Il "suono" è simbolicamente il linguaggio poetico con tutta la sua carica patetica, emotiva e retorica; la "ragione" è quel linguaggio interpretativorazionale che permette alla mente umana non solo la comprensione dell'enunciato poetico, ma anche la derivazione dal suo "suono" di ogni altra verità e filosofica e morale.

La ricerca di quel "segno" che è il volgare illustre procede su due fronti: uno empirico e l'altro deduttivo. Il "fallimento" della ricerca empirica (1.16.1) non significa però la fine della ricerca di quella "pantera profumata" che è il volgare illustre. Continuando la sua ricerca con "strumenti più razionali" (1.16.1), Dante impiega il metodo deduttivo per trovare "quella cosa" con la quale tutte le cose di uno stesso genere possono essere "confrontate e valutate e che serve anche da misura" (1.16.2). Per Dante, ogni cosa è "mensurabile [...] secundum quod in genere est, illo quod semplicissimum est in ipso genere" (1.16.3) ["ogni cosa, insomma, è misurabile, in quanto appartenente a un dato genere, secondo l'elemento più semplice che è in quel genere"]. Nel cercare il volgare illustre Dante trova che questo elemento più semplice, l'elemento che è la misura di tutti i volgari locali degli Italiani, appartiene a "ogni città italiana ma non sembra appartenere a nessuna" (1.16.6). Nello scoprire che il volgare illustre esiste in tutti i luoghi e nello stesso tempo in nessun luogo, Dante raggiunge il limite del paradosso: l'universale linguistico che egli cerca si manifesta come astratto e inconsistente nello stesso momento in cui dimostra di essere concreto, reale e empiricamente misurabile. " Il volgare illustre non è la lingua di nessun luogo reale; si manifesta invece come la lingua della sapienza civile in tutte le sue qualità poetiche e retoriche più alte:

Per hoc quoque quod illustre dicimus, intelligimus quid illuminans et illuminatum prefulgens: et hoc modo viros appellamus illustres, vel quia potestate illuminati alios et iustitia et karitate illuminant, vel quia excellenter magistrati excellenter magistrent, ut Seneca et Numa Pompilius. Et vulgare de quo loquimur et sublimatum est magistratu et potestate, et suos honore sublimat et gloria. (1.17.2)

[Con illustre si vuol intendere ciò che illumina e, se illuminato, risplende; così, si dicono illustri gli uomini o perché, illuminati dal potere, diffondono sugli altri luce di giustizia e carità o perché, depositari di un alto magistero, perfettamente insegnano, come Seneca e Numa Pompilio. E il volgare di cui stiamo parlando è eccellente per magistero e potere e innalza i suoi con onore e gloria. $]^{12}$

La lingua della nutrice è anche quella vera lingua poetica e retorica da cui derivano le più alte verità morali e filosofiche della polis: è la materia prima di quel vero linguaggio poetico in cui ogni società umana 'trova', proprio nel senso provenzale di trobar, i fondamenti concreti del suo sapere collettivo. 
È proprio questo volgare poetico, fonte di ogni verità umana primordiale e concreta, che viene rifiutato da Ulisse e Diomede nel Canto 26 dell'Inferno. La scissione comunicativa fra Dante e gli eroi greci quindi non è dovuta tanto a quel contrasto fra poesia epica dello stile alto e poesia umile della comedía cristiana, anche se questo contrasto è chiaramente presente nell'episodio; la scissione è dovuta piuttosto al contrasto fra due modi diversi di intendere la natura della sapienza umana e le sue radici primordiali.

Se nel mondo della Commedia Virgilio è escluso dal Paradiso perché non è cristiano - "nacqui sub Iulio [...] nel tempo de li dei falsi e bugiardi" (Inf. 1.70-72) $)^{13}$ - egli rimane tuttavia poeta epico per eccellenza della tradizione latina: e questo non solo perché aveva cantato la fondazione di Roma ma anche perché aveva insegnato e quindi trasmesso a Stazio la natura della vera lingua poetica. È vero che Virgilio è per Stazio il modello ideale del poeta non soltanto quale strumento di trasformazione morale e spirituale, ma anche quale profeta del cristianesimo; egli lo celebra come tale in Purg. 22:

Facesti come quei che va di notte,

che porta il lume dietro e sé non giova,

ma dopo sé fa le persone dotte,

quando dicesti: "Secol si rinova;

torna giustizia e primo tempo umano,

e progenie scende da ciel nova". $(67-72)^{14}$

Ma Virgilio è anche colui che trasmette attraverso la sua lingua poetica la lingua comune della "mamma" e della "nutrice":

Al mio ardor fuor seme le faville, che mi scaldar, de la divina fiamma onde sono allumati piú di mille; de l'Eneïda dico, la qual mamma fummi, e fummi nutrice, poetando: sanz' essa non fermai peso di dramma. (Purg. 21.94-99)

L'insegnamento di Virgilio ha per Stazio un doppio effetto - "Per te poeta fui, per te cristiano" (Purg. 22.73) $)^{15}$ - proprio perché la rivelazione delle piú alte verità religiose e morali di una intera comunità vengono trasmesse poeticamente attraverso la lingua primordiale della nutrice. ${ }^{16}$ Se però un legame linguistico-poetico esiste fra Virgilio e Stazio, non segue affatto che uno stesso le-game esista fra Virgilio e Dante. ${ }^{17}$

Il primo incontro fra Virgilio e Dante ha luogo nel Canto 1 dell'Inferno: 
rispuos' io lui con vergognosa fronte.

"O de li altri poeti onore e lume, vagliami " 1 lungo studio e "l grande amore che $m$ 'ha fatto cercar lo tuo volume.

Tu se' lo mio maestro e 'l mio autore, tu se' solo colui da cu' io tolsi

lo bello stilo che m'ha fatto onore". (79-87)

Se Dante riesce a comprendere Virgilio solo dopo "il lungo studio", il "volume" virgiliano - l'Eneide - non può contenere la sua vera lingua natia: Virgilio, sebbene "fonte che spande di parlar sí largo fiume", è il poeta della grammatica, della lingua dei pochi. Tramite la figura di Virgilio, la rottura fra Dante e Ulisse diventa chiara: Virgilio è sempre quel poeta che scrisse "li alti versi" (Inf. 26.82) del mondo classico, quei versi in cui vengono cantate le imprese di Ulisse e Diomede. ${ }^{18}$ Sono i versi che Dante apprende solo studiando e non attraverso l'insegnamento diretto della nutrice. Ecco perché è significativa la divisione dell'episodio di Ulisse in due parti: una in cui Virgilio elenca $i$ peccati di Ulisse e Diomede; un'altra in cui Ulisse racconta il suo "folle volo".

La prima parte dell'episodio di Ulisse interessa soprattutto Virgilio, il poeta della fondazione di Roma. L'elenco dei peccati di Ulisse e Diomede ${ }^{19} \mathrm{è}$ un riassunto in miniatura dell'opera epica virgiliana:

\section{"Là dentro si martira}

Ulisse e Diomede, e così insieme a la vendetta vanno come a l'ira; e dentro da la lor fiamma si geme l'agguato del caval che fé la porta onde uscì de' Romani il gentil seme.

Piangevisi entro l'arte per che, morta,

Deïdamìa ancor si duol d'Achille, e del Palladio pena vi si porta". (Inf. 26.55-63)

I tre peccati qui elencati sono tutti contra Troia e quindi contra Roma e il suo "gentil seme". Virgilio implicitamente ricorda il ruolo che la sua opera poetica ha nel cantare un'episodio altamente significativo per lo svolgersi della storia sacra cristiana: la caduta di Troia. Il poeta di Mantova non puo fare altro che compiacersi, forse anche con dolore e nostalgia, della potenza dei suoi "alti versi" nel rievocare le empie imprese di Ulisse e Diomede. A Dante pellegrino, invece, interessa sapere soprattutto dove l'Itacense "a morir gissi" (Inf. 26.84): il "folle volo" è l'episodio che giustamente appartiene a Dante, all'uomo medievale alla ricerca di una propria storia epica e di una propria lingua con la quale scriverla. Se Virgilio guarda indietro a una storia epica già completa e tutta nel passato, Dante guarda verso una storia epica non ancora scritta, e in una lingua poetica non ancora trovata. ${ }^{20}$ Visto così, 
il viaggio di Ulisse rappresenta il tentativo fallito di andare oltre l'epica classica, di continuare, cioè, la sua storia - proprio quella ricerca di "virtute e canoscenza" (Inf. 26.120) - nel "mondo sanza gente" (Inf. 26.117) ${ }^{21}$

Il Canto 26 dell'Inferno va necessariamente letto insieme al Canto 26 del Purgatorio e al Canto 26 del Paradiso. In tutti e tre questi canti incontriamo personaggi - Ulisse, Arnaut Daniel, ${ }^{22}$ Adamo - avvolti dal fuoco. La metafora della fiamma ${ }^{23}$ lega inestricabilmente questi tre episodi: ognuno di essi pone un problema linguistico, ${ }^{24} \mathrm{e}$ rappresenta in qualche modo o l'inizio o il culmine di una tradizione o avvenimento storico. Arnaut Daniel, il "miglior fabbro del parlar materno" che in "versi d'amore e prose di romanzi / soverchiò tutti" (Purg. 26.117-19), rappresenta il culmine di una tradizione poetica, linguistica e culturale. ${ }^{25}$ L'evoluzione poetica di Arnaut è paragonabile a quella di Dante, però con questa differenza: se Dante rappresenta anche lui come Arnaut il culmine di una tradizione, egli rappresenta anche il tentativo di andare oltre i limiti di questa tradizione. Dopo il suo discorso in provenzale alla fine di Purgatorio 26 (139-47). Amaut "s'ascose nel foco che li affina" (Purg. 26.148); Dante invece continua il suo viaggio alla ricerca di quel linguaggio poetico illustre, universale e comune - continua, cioè, la scrittura della massima espressione poetica di una intera comunità, l'epica. ${ }^{26}$

Se la ricerca linguistica e poetica di Dante è paragonabile al viaggio di Ulisse - entrambi alla ricerca in qualche modo della sapienza in generale possiamo cominciare a specificare il modo in cui i due viaggi divergono. La teoria linguistica definitiva di Dante viene espressa attraverso la figura di Adamo, il terzo personaggio di quella triade che parla attraverso le fiamme:

"La lingua ch'io parlai fu tutta spenta
innanzi che a l'ovra inconsummabile
fosse la gente di Nembròt attenta:
ché nullo effetto mai razionabile,
per lo piacere uman che rinovella
seguendo il cielo, sempre fu durabile.
Opera naturale è ch uom favella;
ma così o così, natura lascia
poi fare a voi secondo che v'abbella.
Pria ch'i' scendessi a l'infernale ambascia,
Is'appellava in terra il sommo bene
onde vien la letizia che mi fascia;
e $E l$ si chiamò poi: e ciò convene,
ché l'uso d'i mortali è come fronda
in ramo, che sen va e altra vene". ( $P$ ar. 26.124-138)

Adamo modifica in questi versi la teoria linguistica che Dante aveva elaborato nel De Vulgari Eloquentia: ${ }^{27}$ non esiste più nel Canto 26 del Paradiso una lingua divina che partendo dal primo uomo resiste a ogni mutamento 
e diventa poi la lingua ideale di Cristo (De Vulgari Eloquentia 1.4.4-6). L'unica lingua veramente nobile che rimane per Dante è quella concreta e naturale della nutrice: la lingua umana rimane sostanzialmente contingente e dipendente da quel primo 'suono' che cerca di rinnovare, con ogni nuova epoca storica umana, la gioia della creazione divina. È l'uomo stesso, dunque, che crea la propria lingua, il proprio enunciato poetico, nel momento in cui egli risponde alla lingua divina espressasi attraverso la natura (De Vulgari Eloquentia 1.4.4-7). Non esiste per Dante né una lingua ideale e astratta, né una grammatica universale a priori ${ }^{28}$ Il volgare illustre è sempre quell' 'universale concreto' legato alla nutrice e quindi simbolicamente alla comunità umana. Dante non cerca nessuna lingua né universale né astratta; egli cerca invece la possibilità dell'espressione pienamente poetica di quella gioia che Adamo prima della caduta aveva provato davanti alla creazione divina:

Quid autem prius vox primi loquentis sonaverit, viro sane mentis in promptu esse non titubo ipsum fuisse quod "Deus" est, scilicet $E l$, vel per modum interrogationis vel per modum responsionis. Absurdum atque rationi videtur orrificum ante Deum ab homine quicquam nominatum fuisse, cum ab ipso et in ipsum factus fuisset homo. Nam sicut post prevaricationem humani generis quilibet exordium sue locutionis incipit ab "heu", rationabile est quod ante qui fuit inciperet a gaudio; et cum nullum gaudium sit extra Deum, sed totum in Deo, et ipse Deus totus sit gaudium, consequens est quod primus loquens primo et ante omnia dixisset "Deus". (1.4.4)

[Che cosa poi la voce del primo parlante abbia detto per la prima volta, non dubito che a ogni uomo saggio venga subito in mente essersi trattato di "Dio", ovvero $E l$, in forma di domanda o di risposta. Sembra assurdo e irrazionale che dall'uomo sia stato nominato qualcos'altro prima di Dio, poiché da Lui e per Lui fu creato. Infatti, come, dopo la ribellione dell'umano genere, ognuno comincia a parlare con un "ahi", così è ragionevole che le parole dette prima comincino dalla Gioia; e poiché non vi è alcuna gioia fuori di Dio, ma tutta è in Dio e lo stesso Dio è tutta gioia, ne segue che il primo parlante per prima cosa abbia detto innanzitutto "Dio".]

La ricerca della lingua poetica comprende la ricerca del signum nella sua complessità doppia di "suono" e ragione. Dante è il poeta della sapienza che parte dai sensi, dal "suono" poetico. Il "pan de li angeli" è il discorso secondario, il commento filosofico-razionale che spiega ciò che la sapienza poetica rivela ai sensi umani. ${ }^{29} \mathrm{E}$ il lato razionale del signum dantesco che segue l'atto creativo poetico e rende completo ogni atto di comunicazione umana. Ulisse non parla con Dante non perché non comprende il volgare ma perché lo disprezza: Ulisse, insomma, disprezza la lingua primordiale adamitica attraverso la quale il primo uomo aveva espresso la sua gioia di fronte alla natura divina e all'esistenza. Egli preferisce invece la grammatica, la lingua regolata, la lingua delle astrazioni razionali e vuote che non sono fondate sul concreto di quel linguaggio poetico comune e illustre..$^{30}$ Le astrazioni sim- 
boliche di Ulisse - egli cerco il "mondo sanza gente" (Inf. 26.117) - non possono avere né sostan-za attuale né valore gnoseologico in quanto non derivano dai veri principi primordiali della conoscenza umana, cioè da quelle verità poetiche comuni a tutti. Dalla vera lingua poetica, la lingua primordiale della nutrice, nasce ogni altro ordine di verità e di conoscenza umana: il discorso poetico precede ogni altro tipo di discorso o di linguaggio, e ogni altro tipo di conoscenza. ${ }^{31}$

La lingua di Ulisse è il signum dantesco senza il lato sensibile, il lato del "suono" e quindi della poesia. Nel lasciare la sua comunità, Ulisse simbolicamente lascia la vera radice o il vero fondamento di ogni conoscenza umana: la poesia, ovvero quel vero linguaggio poetico che ci viene insegnato spontaneamente dalla nutrice e in cui il primo uomo, Adamo, aveva reso gloria alla mente divina. Il peccato di Ulisse ha quindi significato teologico non solo nel senso cristiano, ma anche nel senso poetico-epistemologico: Ulisse compie un errore nel volere trovare il principio primordiale della conoscenza umana nel segno senza "suono", privo di poesia. Egli vuole stabilire ogni conoscenza umana invece nel signum puramente razionale, astratto e lontano da ogni sostanza concreta, umana. Il "folle volo" di Ulisse quindi esprime simbolicamente l'impossibilità di basare la conoscenza umana sull'universale astratto e solo razionale. L'unica vera base di conoscenza umana rimane quel paradossale "universale concreto" del volgare che è la materia grezza del volgare illustre dal quale viene plasmato quel signum che rende possibile ogni vera conoscenza umana: il signum in cui il "suono" (cioè la poesia) precede la ragione, e in cui l'atto poetico e l'atto interpretativo/contemplativo coesistono armonicamente.

Ed ecco perché, finalmente, Arnaut Daniel viene celebrato nel Canto 26 del Purgatorio: è il poeta per eccellenza non solo del "parlar materno", della lingua primordiale della nutrice, ma anche del "suono". Il trobar clus si $^{32}$ Arnaut Daniel rende evidente l'importanza del "suono", della poesia non solo come espressione del pensiero umano o della condizione esistenziale dell'io, ma anche come nesso enunciativo primordiale che precede ogni altro tipo di discorso umano, sia razionale che prosastico.

University of Toronto

\section{NOTE}

1 Nel suo saggio ormai famoso, "La tragedia di Ulisse", Bruno Nardi scrive che "nel folle volo di Ulisse Dante scorge una continuazione del peccato originale" (14). Il Nardi vede in Ulisse la personificazione teologicamente negativa della "ragione umana insofferente di limiti e ribelle al decreto divino che interdiceva all'uomo di mettersi sulla via che conduce al legno della vita. Nella follia di Ulisse e dei suoi compagni v'è tutto l'orgoglio umano che spinse Adamo ed Eva a gustare il frutto della scienza del bene e del male, per essere simili a Dio; v`è anzi lo stesso orgoglio di Lucifero" (13-14). 
2 Cfr. Mario Fubini: "Non è simile a un Dio ribelle né è un superuomo l'Ulisse di Dante, come tenderebbero talora di raffigurarlo certi lettori modemi" (466). Cfr. anche Fubini, $1 /$ peccato 1 36.

3 Per il dibattito Nardi-Fubini e la posizione del Montano, vedi Thompson (33-35); cfr. anche Barolini (49-51).

4 Altri studi fondamentali sul canto di Ulisse sono quelli di Padoan, Forti, Avalle, Mariano, Sturm, Mazzotta ("Poetics"), Pertile e Bommarito.

5 Anche lannucci interpreta questo contrasto drammatico soprattutto in termini stilistici e di registro linguistico: "l' arrog anza dei greci, alla quale Virgilio fa appello per giustificare il suo intervento, è dovuta proprio al disdegno e all'intolleranza che essi provano verso coloro che non si conformano al loro codice stilistico, il quale a sua volta riflette un ideale di cultura. Peri greci è proprio la lingua ciò che distingue l'uomo dall'animale, ed è il livello di articolazione del linguaggio e la sua razionalità che determinano la superiontà tra gli uomini" ("Il 'folle volo' di Ulisse" 155). Sull'importanza per Dante dei vari livelli di registro linguistico-stilistico e sul significato del termine comedia adoperato come categoria letteraria per descrivere la natura del poema dantesco, si veda Baranski (225-29; 237-38). Si veda anche Picone (121).

6 Cito dall'edizione di Mengaldo; le traduzioni italiane invece sono quelle di Coletti.

7 Il problema del significato di "grammatica" è uno dei punti più discussi della critica dantesca; rinvio agli studi più importanti su questo tema: Corti (Dante), Lo Piparo, Tavoni, Mengaldo ("Un contributo"). L'idea di grammatica che intendo qui, comunque, deriva soprattutto dalle idee di Emesto Grassi, il quale scrive che Dante "discems two kinds of language. The first kind is ahistorical and artificial, a language in which the different languages of the different peoples has been crystallized, so to speak, according to fixed rules. This for Dante is the universal language Latin. He also calls this language "grammatical' because in his view it is constructed in a purely rational way and so is ahistorical" (77). Fra i poeti classici denominati "regolati" da Dante sono inclusi Virgilio, Ovidio delle Metamorfosi, Stazio e Lucano (2.6.7). Sul ruolo di Stazio nellaDe Vulgari Eloquentia e nella Commedia, cfr. Iannucci, "Dante" 32-33.

8 Cfr. Grassi: "[Dante] claims that "true," "authentic' language can never be 'artificial' or "fixed" (he is referring to Latin) but only one in which men work, act, and live-that language in which they express their strivings and passions that stem from a concrete situation. He means that second kind of language, the particular people's native tongue and all its historicity" (78).

9 Per lingua intendo non tanto quella capacità della mente umana matura di esprimere in modo logicamente razionale un suo pensiero quanto il suono concreto del bambino che l'uomo riesce poi a plasmare e a trasformare artisticamente in poesia.

10 Il signum dantesco è significativo per l'episodio di Ulisse perché l'eroe greco è proprio colui che, come Adamo che ha voluto "trapassar il segno" (Par. 26.117), è andato oltre quelle colonne "dove Ercole segnò i suoi riguardi" (Inf. 26.108). Se il concetto del signum è legato in qualche modo all'idea del limite, bisogna vedere in che modo e con quale significato i due concetti sono messi insieme.

11 Cfr. Grassi: "The 'authenticity" of the language that the poet as vates or "orator' realizes can be found and identified only in the multiplicity of different dialects that are the expression of a particular historical situation" (78). Cfr. anche Franco Lo Piparo, il quale, criticando la teoria linguistica dei modisti come Boezio di Dacia che vede la grammatica quale entità esistente " $a b$ eterno nella mente umana", afferma la priorità del volgare: "In Dante ad essere naturali e universali sono idiomi volgari; le grammatiche sono idiomi ausiliari artificialmente costruiti che si aggiungono ai primi" (19).

12 Grassi: "Illustre is the first characteristic of the language that Dante seeks and wants to realize in his work because of the political and historical situation in which he lives. Illustre could be translated as 'famous' or 'exalted,' but I believe that these adjectives do not express the quality that Dante has in mind. This requires that the verb be rendered illustrare, as 'illuminating' in the sense that language brings men to an insight into higher truths and the movements of the soul by virtue of the 'illuminating brilliance' of its creative poetic power. In other words genuine language must stem from the depths of human creativity, be directed to all aspects of humanity, and lead to spir- 
itual and practical self-realization. Only in this way can it also embrace and advance men's political life as well" (81). Per gli altri attributi del volgare illustre - cardinale, curiale, aulica - vedi Grassi (81-82).

13 L'edizione della Divina Commedia citata qui è quella a cura di Giorgio Petrocchi.

14 Questi versi richiamano il brano del De Vulgari Eloquentia, 1.1.1, citato sopra, in cui Dante critica coloro che "credono di avere davanti ciò che invece hanno dietro".

15 Per lannucci l'episodio di Stazio nel Purgatorio "appartiene più a Virgilio che a Stazio" e "drammatizza sia il valore esemplare di Virgilio e della sua opera che i suoi limiti tragici" ("Dante" 33). Se Virgilio dà un modello formale da seguire, egli non è in grado di riempirlo con un contenuto comico-cristiano, un contenuto che tratti "la dannazione o la salvezza dell 'anima cristiana" ("Dante" 35). Secondo Picone, inoltre, Stazio risponde all 'esigenza di "affabulare [...] il passaggio delle consegne poetiche del mondo classico a quello cristiano, e di presentare quindi la prima figura di poeta modemo: categoria alla quale Dante appartiene" (114). Cfr. anche lannucci, "La Divina Commedia" (40-41).

16 Nel Purgatorio 22 Virgilio chiama le Muse "le nutrice" dei poeti:

"Costoro [Terenzio, Cecilio, Plauto, Varro] e Persio e io e altri assai"

rispuose il duca mio "siam con quel greco [Omero]

che le Muse lattar piú ch'altri mai,

nel primo cinghio del carcere cieco:

spesse fiate ragioniam del monte

che sempre ha le nutrice nostre seco". (Purg. 22.100-5)

La nutrice viene menzionata per la terza e ultima volta nel canto XII del Paradiso: secondo il racconto di San Bonaventura, essa è spesso presente alle preghiere di San Domenico: "Spesse fiate fu tacito e desto/trovato in terra da la sua nutrice, / come dicesse: 'Io son venuto a questo' " (Par. 12.76-78). La nutrice è quindi sempre legata in qualche modo a grandi "imprese" poetico-religiose.

17 Cfr. Picone: "Il canone degli auctores, proposto nella Commedia, metterà [...] l'enfasi proprio sull'incompletezza 'tragica' del mondo antico: incompletezza che si manifesta non solo nella condanna di un personaggio come Ulisse, ma anche nell' incapacità di Virgilio di guidare Dante oltre un certo limite di conoscenza umana e terrena" (114).

18 Cfr. Iannucci: "Qui nelle malebolge, come nel Limbo, Virgilio si pone fra [Dante] ed Omero e media lo spazio fra l"epica greca e la nuova epica cristiana che viene creata in questo momento" ("Dante" 26). Inoltre, in Inferno 26 Dante si misura non solo "con Omero stesso" ma "anche con Virgilio", e se nel resto della Commedia domina lo stile cristiano misto mentre in Inferno 26 domina lo stile alto e "tragico" di Omero e Virgilio, Dante tuttavia li "supera certo [...] in inventiva, e forse persino nello stile, ma di nuovo egli grida vittoria soprattutto a ragione del significato superiore dell 'episodio. In Inferno 26, il soggetto, lo stile e il tono sono classici, ma il significato è profondamente cristiano" ("Dante" 34-35).

19 Anche il problema del vero peccato di Ulisse è stato molto dibattuto dalla critica dantesca: cfr., per esempio, Fubini (465). Per un 'ottima trattazione del problema, si veda il saggio di Anna Hatcher. Su questo argomento, cfr. anche Cassell (85-95).

$20 \mathrm{Cfr}$. Hollander: "If the use of the Aeneid and Dante's own words point us back, the Ulysses episode also points us forward" (122).

21 Barolini invece identifica in termini positivi l'impresa ulisseana con quella di Dante poeta: "If the pilgrim leams to be not like Ulysses, the poet is conscious of having to be ever more like him. The Paradiso, if it is to exist at all, cannot fail to be transgressive; its poet cannot fail to be a Ulysses, since only a trapassar del segno will be able to render the experience of trasumanar" (54). Se nel Paradiso "significar per verba / non si poria," il poeta deve "trapassare" ogni segno: "only a trespass of the sign can render an experience for which no signs are sufficient" (55). Inoltre, "Ulysses is designed as a recurring presence because the issue of the trapassar del segno, of Adam's sin conceived not literally as the eating of the tree but metaphorically as a transgression, is one that Dante cannot discount [...]. No matter how orthodox his theology (and it is not so orthodox), no matter how fervently Dante believes in and claims the status of true prophet, of directly 
inspired poet, of scriba Dei, the very fiber of the Commedia consists in going beyond" (58).

22 Per le poesie di Amaut, cfr. le edizioni a cura di Gianluigi Toja e a cura di Maurizio Perugi.

23 Su la metafora della fiamma, cfr. il saggio della Corti ("On the metaphors of sailing").

24 Non dimentichiamo, inoltre, che l'inizio del discorso provenzale di Arnaut - "Tan m'abellis vostre cortes deman" (Purg. 26.140) - richiama il discorso di Adamo sulla lingua umana: "natura lascia / poi fare a voi secondo che v'abbella" (Par. 26.130-132). Cfr. il commento di Natalino Sapegno al Purgatorio (295).

25 Sull'importanza di Amaut per Dante, si veda Barolini, Dante's Poets (112-14: 176-79). Per Dante, secondo la Barolini, Amaut rappresenta "novità" ("newness") nel campo della lirica amorosa: "Of all the troubador claims for newness, only Amaut's is - for Dante - invested with the epistemological guarantee to legitimize his "chantar tot nou' ("wholly new song'); only Amaut's poetry, therefore, can be considered a true precursor of the 'nove rime' " (Dante's Poets 114). Cfr. anche la voce del Viscardi nell'Enciclopedia dantesca.

26 Per Barolini l'andare oltre la lirica amorosa, la cui tradizione vede il suo culmine in Amaut, significa fondere insieme poesia amorosa e poesia politica: "The fusion between love poetry and political poetry [...] alone can make a poet truly great [...] such a fusion seems to be the sine qua non for surpassing what Dante sees as the lyric stage of poetic development" (Dante's Poets 184). Inoltre, per Dante "only epic poets [...] combine eros with commitment, fusing the search for salus and venus into the search for virtus, and thereby becoming a poet of rectitude" (187), ovvero un poeta che parla per un'intera società e che dà vita a una poesia "charged with the moral and political militancy required to preserve ethical life for society as a whole" (186).

27 Lo Piparo vede la teoria linguistica espressa qui come estensione delle idee già presenti nel $D e$ Vulgari Eloquentia (22). Secondo Lo Piparo, il signum che Dante teorizza nel De Vulgari Eloquentia è mutabile non solo sul piano sensuale ma anche sul piano razionale, e "un segno linguistico siffatto possiede [...] una intrinseca capacità di mobilità semantico-razionale del tutto inedita nella linguistica medievale". La teoria di Adamo, dunque, è "la continuazione dei due capitoli semiologici del De Vulgari Eloquentia" (25). Adamo, inoltre, non fa risalire la causa del mutamento linguistico "al segno in quanto sonus $\mathrm{ma}$ in quanto razionale. Il mutamento è effetto razionale: ché nullo effetto mai razionabile/ [...] sempre fu durabile" (25-26). Questa teoria del signum contrasta con quella dei modisti, i quali vedono la varietà delle lingue come fatto puramente fonico, superficiale e accidentale, e quindi come aspetto solo del signum-vox (27).

28 Maria Corti parla invece di una forma locutionis che, "concreata con l'anima dell'uomo, non è una lingua concreta, bensì la 'causa formale' e il principio generale strutturante della lingua, sia per quanto riguarderà il lessico sia per quanto riguarderà i fenomeni morfosintattici della lingua che Adamo lentamente fabbricherà, vivendo e nominando le cose" (Dante 47).

29 Ovviamente la questione sia dell'esegesi allegorica sia di quell'arte poetica che si basa proprio sulle strutture dell'arte esegetica medioevale è molto complessa, come è il problema dell' allegoria come la troviamo in Dante nei suoi vari scritti quale il Convivio e la lettera a Can Grande della Scala. Rinvio a Charity, Hollander, Pepin e Singleton. Qui è sufficiente indicare la ricerca di una vera lingua poetica volgare che è fonte di ogni vera conoscenza umana come tema che rientra nel dramma del contrasto fra Dante e Ulisse.

30 Mazzotta scrive che il viaggio di Ulisse, e soprattutto la sua orazion picciola, indica una scissione fra res e signa, fra realtà e immagine, in cui "language seems to originate in the void, in a condition of total separation from the world of reality, where it makes seductive promises to produce experience and is doomed to failure" ("Poetics" 39).

31 Cfr. l'introduzione di Mazzotta al suo libro Dante's Vision (3-14).

32 Per il trobar clus di Amaut, cfr. Toja.

\section{OPERE CITATE}

Alighieri, Dante. Convivio. Ed. Piero Cudini. Milano: Garzanti, 1980.

. De Vulgari Eloquentia. Ed. Pier Vincenzo Mengaldo. Opere minori. 2 voll. MilanoNapoli: Ricciardi, 1979. 


\section{De Vulgari Eloquentia. Ed. Vittorio Coletti. Milano: Garzanti, 1991. \\ La Commedia secondo l'antica vulgata. Ed. Giorgio Petrocchi. 2 ed. 4 voll. Firenze: Le Lettere, 1994. \\ 1956. \\ _- "Epistola X: To Can Grande della Scala". The Letters of Dante. Ed. Paget Toynbee. Oxford: Oxford UP, 1920. 160-211.}

Avalle, D’Arco Silvio. "L'ultimo viaggio di Ulisse". Studi danteschi 43 (1966): 35-67.

Baranski, Sygmunt. "Dante e la tradizione comica latina". Dante e la "bella scola". 225-45.

Barolini, Teodolinda. Dante's Poets. Textuality and Truth in the Comedy. Princeton: Princeton UP. 1984.

-The Undivine Comedy. Detheologizing Dante. Princeton: Princeton UP, 1992.

Bommarito, Domenico. "Il mito di Ulisse e la sua allegorizzazione in Boezio e Dante. Ulisse: il tema dell"homo sapiens"”. Forum italicum 17 (1983): 64-81.

Cassel, Anthony K. "Ulysses". Dante's Fearful Art of Justice. Toronto: U of Toronto P, 1984. 85-95.

Charity, Allan C. Events and their Afterlife. The Dialectics of Christian Typology in the Bible and Dante. Cambridge: Cambridge UP, 1966. 167-256.

Corti, Maria. Dante a un nuovo crocevia. Firenze: Sansoni, 1981.

. "On the Metaphors of Sailing, Flight and Tongues of Fire in the Episode of Ulysses (Inferno 26)". Stanford Italian Review 9 (1990): 33-47.

Daniel, Arnaut. Canzoni. Ed. Gianluigi Toja. Firenze: Sansoni, 1960. 1978.

Forti, Fiorenzo. "Ulisse”. Cultura e scuola 13-14 (1965): 499-517.

Freccero, John. "Dante's Ulysses: From Epic to Novel". Dante: The Poetics of Conversion. Cambridge: Harvard UP. 136-51.

Fubini, Mario. "Il peccato di Ulisse". Belfagor 2 (1947): 461-75.

Ill peccato di Ulisse e altri scritti danteschi. Milano-Napoli: Ricciardi, 1966.

Grassi, Emesto. Rhetoric as Philosophy. University Park: Pennsylvania State UP, 1980.

Hatcher, Anna. "Dante, Ulysses and Guido da Montefeltro". Dante Studies 88 (1970): 109-17.

Hollander, Robert. Allegory in Dante's Commedia. Princeton: Princeton UP, 1969.

Iannucci, Amilcare A. "Dante e la "bella scola' della poesia (Inf. 4.64-105)". Dante e la "bella scola". 19-37.

—_. "Il 'folle volo' di Ulisse: il peso della storia". Forma ed evento nella Divina Commedia.

Roma: Bulzoni, 1984. 145-88.

- "La Divina Commedia: teoria e prassi dei generi letterari". Forma ed evento. 13-50.

Dante e la "bella scola" della poesia. Autorità e sfida poetica. Ed. Amilcare A. Iannucci. Ravenna; Longo, 1993.

Lo Piparo, Franco. "Dante linguista anti-modista". Italia linguistica: idee, storia, strutture. Ed. Federico Albano Leoni, Daniele Gambarara, Franco Lo Piparo, Raffaele Simone. Bologna: Il Mulino, 1983. 9-30.

Mariano, Emilio. "Canto XXVI". Lectura dantis scaligera: Inferno. Firenze: Le Monnier, 1967.

Mazzotta, Giuseppe. "Poetics of History." Diacritics 5 (1975): 37-44.

-Dante's Vision and the Circle of Knowledge. Princeton: Princeton UP, 1993.

Mengaldo, Pier Vincenzo. "Un contributo all'interpretazione di 'De vulgari eloquentia', I, i-ix," Belfagor 44 (1989): 539-58.

Montano, Rocco. "Il 'folle volo' di Ulisse". Suggerimenti per una lettura di Dante. Napoli: Conte, 1956. 131-90.

Nardi, Bruno. "La tragedia di Ulisse". Studi danteschi 20 (1937): 5-15.

Padoan, Giorgio. "Ulisse 'fandi fictor' e le vie della sapienza". Studi danteschi 37 (1960): 21-61.

Pepin. Jean. Dante e la tradition de l'allégorie. Montréal: Institut d'études médiévale, 1970.

Pertile, Lino. "Dante e l'ingegno di Ulisse". Stanford Italian Review 1 (1979): 35-65.

Picone, Michelangelo. "L'Ovidio di Dante". Dante e la "bella scola". 107-44. 
Singleton, Charles S. Dante Studies I: "Commedia" : Element's of Structure. Cambridge: Harvard UP, 1954.

Sturm, Sara. "Structure and Meaning in Inferno XXVI". Dante Studies 92 (1974): 93-106.

Tavoni, Mirko. "Contributo all'interpretazione di 'De vulgari eloquentia' I, 1-9." Rivista di letteratura italiana 5 (1987): 385-453.

Thompson, David. "Dante's Ulysses and the Allegorical Journey". Dante Studies 85 (1967): 33-58.

Toja, Gianluigi. "Trobar clus". Enciclopedia dantesca. 6 voll. Roma: Istituto dell'enciclopedia italiana, 1970-1978. 5.383-87.

Viscardi, Antonio. “Amaut Daniel”. Enciclopedia dantesca. 1.732-36. 